\title{
The Role of Reading Order and Monitoring Skills During Hypertext Comprehension
}

\author{
Mari Carmen Puerta Melguizo ${ }^{*}, 1$, Ladislao Salmerón ${ }^{2}$, R. Ignacio Madrid ${ }^{3}$ and \\ Herre van Oostendorp ${ }^{1}$
}

${ }^{I}$ Center for Content and Knowledge Engineering, Institute of Information and Computing Sciences, Utrecht University, The Netherlands

${ }^{2}$ Department of Developmental and Educational Psychology, University of Valencia, Spain

${ }^{3}$ Department of Experimental Psychology and Physiology of Behaviour, University of Granada, Spain

\begin{abstract}
In this paper we discuss two experiments on the role that comprehension monitoring skills play in selecting the reading order of hypertextual information and comprehending this information. In the first experiment, learners who could be characterized as learners with low prior knowledge comprehended the hypertext better when they selected a coherent reading order. Further, number of links per page had no effect on cognitive load or comprehension. Finally, link support enhanced comprehension. In the second experiment, we showed that low prior knowledge learners are able to select a high coherent reading order strategy provided that these learners have high monitoring skills with regard to their comprehension process. We conclude that an important characteristic of hypertext comprehension is the skill to monitor the level of coherence and regulating the reading order is in this respect essential.
\end{abstract}

\section{INTRODUCTION}

The main characteristic of hypertext is that information is presented as a collection of documents containing links and therefore, hypertext allows - or even requires - learners to navigate and sequence information according to their specific needs. Learning with hypertext has become extremely popular in educational settings but still there is not conclusive experimental evidence that probes that learners are better off with hypertexts than with traditional books. This is especially true in the case of learners with low prior knowledge (LPK) on a specific domain. In general, LPK students are more prone to have difficulties with navigation and comprehension of hypertext. For an extensive review see [1].

In relation to comprehension and according to the Construction-Integration model proposed by Kintsch [2], text comprehension consists of forming coherent mental representations from the text during reading. The two most important mental representations are the textbase and the situation model. The textbase is a network-like, mainly hierarchical representation of propositions contained in the text. The situation model is considered the deepest mental representation, and integrates the textbase with the reader's prior knowledge $[2,3]$. It is important to stress here that the most important factors that influence the construction of a situation model are the learner's prior knowledge and the text coherence or the extent to which a reader is able to understand the relations between ideas expressed in a text

*Address correspondence to this author at the Center for Content and Knowledge Engineering, Institute of Information and Computing Sciences, Utrecht University, The Netherlands; E-mail: puerta@cs.uu.nl
[4]. When LPK readers read a highly coherent text they construct better situation models than when they read low coherent ones. For example [5].

Research on hypertext has shown that one important factor that influences comprehension of a hypertext is the reading order readers actively select while navigating in hypertext (for example [6]). A coherent reading order consists of selecting text sections that are semantically related to the one just read. In one experiment [7] found that LPK participants who selected a coherent reading order acquired higher levels of comprehension than participants who selected a less coherent reading order. Readers with more prior knowledge comprehended equally well regardless of the link selection strategy they followed. DeStefano and LeFevre [8] explain this in the following way: every time a reader chooses to follow a link, the text connected to the link can potentially function as an interruption of the ongoing comprehension process. The effects of the interruption in comprehension are minimal if the text connected with the selected link is related to the previous read text. On the other hand, when the selected link leads to a semantically unrelated text, the text coherence decreases and the consequence for the reader is an interruption in the comprehension process. This interruption of the comprehension process particularly affects the development of the situation model [5]. In line with McNamara et al., LPK readers are the ones who particularly experience negative effects of these interruptions. This is indeed our first research question: can we replicate the finding that with LPK readers a low coherent text reading order results in less comprehension?

DeStefano and LeFevre [8] also claim that decisions about which link to follow increase cognitive load. 
According to these authors, hypertexts with more links should produce greater cognitive load than hypertext with few links and this increase in cognitive load can lead to orientation problems and negatively affect comprehension. The second aim of our first experiment is to explore the effects of number of links on cognitive load and comprehension.

On the other hand, several navigation support systems have been proposed to assist LPK learners $[9,10]$. Navigational support has been presented in the form of overviews, concept maps, link suggestions, etc. For example, by providing link suggestions disorientation and cognitive load in hypertext are reduced [11]. Salmerón et al., [10] proposed an automated method for suggesting links based on Latent Semantic Analysis (LSA). By comparing two portions of text with this method one can obtain a measure called LSA cosine that provides a measure of the argument overlap or semantic similarity between the texts [12]. This measure has been used in previous studies for analyzing text coherence in hypertext [6]. Following [10], in the first experiment LSA cosines were used to suggest links to the readers of a hypertext system. In more detail, in the support condition we selected on every page the two links with the highest LSA cosines between the current page content and the link text labels. The third research question is thus whether navigation support is particularly helpful for LPK learners.

Experiment 2 goes one step further: the first study examines the effects of coherence of text reading order; the second study focuses on the cause of a low coherent reading order. It examines more specifically the role of monitoring skills $[13,14]$. Perhaps learners with a low coherent text reading order are characterized by low monitoring skills. In other words the fourth research question is whether monitoring skill is the driving force behind the observed coherence of the text reading order.

\section{EXPERIMENT 1: READING ORDER AND LINK SELECTION}

The main goals of this first study were firstly to explore the effect of reading order that LPK learners selected and secondly to analyze the effects of number of links on cognitive load and comprehension in hypertext. Part of our hypotheses are derived from the predictions of DeStefano and LeFevre [8] who stressed the idea that making navigational choices in a hypertext imposes more cognitive load and affects comprehension specially when the number of links is higher. Thirdly we wanted to test the usefulness of giving navigation support in the form of link suggestions for comprehension, particularly for LPK learners.

\section{Method}

\section{Participants}

Forty-five students from Utrecht University, mainly from Information and Computing Sciences participated in the experiment. Since we were interested in testing our hypotheses on low prior knowledge readers we looked for students that were unfamiliar with the topic presented in hypertext: brain anatomy and functioning. The data of three participants were excluded because they did not follow the instructions properly.

\section{Design and Measures}

An experimental $2 \times 2$ design was used with number of links (3 vs 8 links) and support (no support $v s$ link suggestions) as independent variables. Several dependent variables were measured:

Prior Knowledge (PK). Although we tried to recruit a LPK sample, we tested participants for differences in PK. Prior to the reading phase participants completed a questionnaire with 10 questions reflecting general knowledge about the brain. Questions were extracted from an introductory book on cognitive science [15]. Each question had four choice options, so chance performance was at $25 \%$.

Coherence of the Selected Reading Order. We computed the mean LSA cosines between text transitions for every participant as a semantic measure reflecting text coherence of the reading order selected by the participant. Based on this analysis two groups were distinguished according to the degree of coherence of the reading order they selected during navigation (see below for more details).

Cognitive Load (CL) was measured by a secondary task technique based on the reaction times (RTs) to probe sounds [16]. To obtain participant's RT baseline, at the beginning of the session, they had to react as quickly as possible to 10 beep sounds presented randomly. During the experiment, participants had to press the "z" key as quickly as possible when a beep was presented through the headphones. The data was corrected subtracting the baseline RTs. This measure can be viewed as reflecting the cognitive capacity allocated to the primary task (reading or selecting links). The higher the capacity allocated to the primary task, the longer the reaction times on the secondary task will be. The beeps were presented in a variable interval between 15-45 sec when reading and between 4-9 sec. when selecting links. In our analyses, we used the average CL (during selection as well as during reading) reflecting the intensity of the $\mathrm{CL}$ during the tasks.

Comprehension Outcomes. To assess comprehension at a situation model level, we constructed 10 inference questions with four response options. This type of questions required relating information contained in at least two different nodes. The participants completed the questionnaire after reading the hypertext.

\section{Materials}

We used a text about Neuropsychology [17]. The text had 4.440 words and was divided into 21 hypertext pages. The link selection menu was located on the left of the reading area. To test independently cognitive load during text reading and during link selection, the links selection menu was hidden during reading a page and was only shown when participants finished reading that page and pressed a button with the label "I have finished reading". To prevent participants reading twice the same text, links that lead to an already read text were shown in a different color (like visited links in web pages). Participants could click on these links, but a message was then shown telling them that the content was read before and that they had to select a different link.

Using Latent Semantic Analysis (LSA) link labels and page titles were constructed by selecting the most 
representative sentence from each page. For the manipulated links options and the link suggestions in the support condition, LSA cosines were calculated between text contents and the link text labels. To select the links options, on each page the two links with the highest LSA cosines were presented; the rest of the links to complete the menu (until 3 or 8 depending on the condition) was extracted randomly from the pool of link labels. In the support condition the two highest related links were marked with an arrow (>>) near them for making the suggestions. The position order of the links in the menu was randomized.

\section{Procedure}

Participants started the session filling in the prior knowledge questionnaire. Then they completed a detection task to determine their reaction time baseline. After that, the hypertext reading phase started. Participants had to read all hypertext pages and were told that they would receive a comprehension test afterwards. This expectation probably sets in participants a relatively high learning goal. Furthermore they were instructed to select the links that seemed most related to the text just read (in other words, to follow a strategy based on coherence). Simultaneously with reading, participants performed the secondary task whenever they heard a beep through the headphones. The instruction stressed that they had to respond to the sounds as soon as possible, but that reading and comprehending the text were the main tasks. In the conditions where support was presented, it was explained that the system would show an arrow ( $>$ ) near the links that the system assessed as more related with the content just read. When all text contents were read, participants went to the comprehension-testing phase.

\section{Results}

Participants' average scores in the PK were 4.98 (out of 10 ) with a standard deviation of 2.18 . To control the effects of prior knowledge on cognitive load and comprehension outcomes, we included PK as covariate in the analyses. Results with $\mathrm{p}<.05$ were considered significant and marginally significant if $\mathrm{p}$ values were between .05 and .10 .

\section{Coherence of Selected Reading Order}

We divided participants in two groups according to their average text coherence, measured as the mean of the LSA cosines between page transitions. Participants were grouped in a high text reading coherence group $(\mathrm{M}=0.37 ; \mathrm{SD}=0.01)$ and a low text reading coherence group $(\mathrm{M}=0.30 ; \mathrm{SD}=0.04)$, using the median score (Median=0.35) as the cut-off (see [6] for a similar procedure to group reading orders). Table $\mathbf{1}$ shows the distribution of participants in both groups: high versus low coherence of the selected reading order. In the analyses we decided to omit the variable link suggestions because the data set per cell was too small (2) to do proper analyses also on this variable. In a number of separate analyses -not reported here- we did show that links suggestions -compared to no-support - had a significant positive effect on text coherence of the reading order and comprehension (see [18]). Here we focus on the influence of the coherence of reading order on cognitive load and comprehension.
Table 1. Number of Participants Following a High or Low Text Coherence Reading Order by Condition

\begin{tabular}{|c|c|c|c|c|}
\hline \multirow{2}{*}{} & \multicolumn{2}{|c|}{ Low Coherence } & \multicolumn{2}{c|}{ High Coherence } \\
\cline { 2 - 5 } & $\begin{array}{c}\text { No } \\
\text { Support }\end{array}$ & $\begin{array}{c}\text { Link } \\
\text { Suggestions }\end{array}$ & $\begin{array}{c}\text { No } \\
\text { Support }\end{array}$ & $\begin{array}{c}\text { Link } \\
\text { Suggestions }\end{array}$ \\
\hline \hline 3 Links & 6 & 2 & 5 & 8 \\
\hline 8 Links & 8 & 5 & 2 & 6 \\
\hline
\end{tabular}

\section{Cognitive Load}

A main effect of coherence of the selected reading order $(\mathrm{F}(1,37)=11.65 ; p<.01)$ was found on $\mathrm{CL}$ during reading. Participants who followed a more coherent reading order had smaller reaction times $(\mathrm{M}=149 ; \mathrm{SD}=31)$ than subjects who followed a less coherent reading order $(M=200 ; S D=69)$. Regarding CL during link selection, there was a marginally significant main effect of reading order $(\mathrm{F}(1,37)=3.03$; $p=.09$ ). Readers following a low coherence reading order suffered more CL during the link selection process $(\mathrm{M}=262$, $\mathrm{SD}=96)$ than those who followed a high coherence reading order $(M=221, S D=83)$. No significant main effects for number of links and no interaction effects were found (all F's $<1$ ). See Table 2 for details.

Table 2. Average CL (RTs in Milliseconds) Following High or Low Text Coherence Reading Order and Number of Links (Standard Deviation Between Parentheses)

\begin{tabular}{|l|c|c|c|c|}
\hline & \multicolumn{2}{|c|}{ Low Coherence } & \multicolumn{2}{c|}{ High Coherence } \\
\cline { 2 - 5 } & 3 Links & 8 Links & 3 Links & 8 Links \\
\hline \hline Average CL (Reading) & $216(88)$ & $190(55)$ & $155(32)$ & $140(28)$ \\
\hline Average CL (Link selection) & $267(97)$ & $258(99)$ & $236(79)$ & $196(88)$ \\
\hline
\end{tabular}

\section{Comprehension}

We found a marginally significant effect of reading order on inference questions scores $(F(1,37)=3.41 ; p=.07)$, readers following a high text coherence reading order performed better $(\mathrm{M}=4.65 ; \mathrm{SD}=1.84)$ on inference questions than readers following a low text coherence reading order $(\mathrm{M}=3.27 ; \mathrm{SD}=1.96)$. There were no significant effects of number of links or interaction. See Table 3.

Table 3. Average Comprehension Performance Following High or Low Text Coherence Reading Order and Number of Links (Standard Deviation Between Parentheses)

\begin{tabular}{|c|c|c|c|c|}
\hline \multirow{2}{*}{} & \multicolumn{2}{|c|}{ Low Coherence } & \multicolumn{2}{c|}{ High Coherence } \\
\cline { 2 - 5 } & 3 Links & 8 Links & 3 Links & 8 Links \\
\hline \hline Inference \\
questions & $\begin{array}{c}3.50 \\
(1.07)\end{array}$ & $\begin{array}{c}3.31 \\
(1.84)\end{array}$ & $\begin{array}{c}3.85 \\
(2.19)\end{array}$ & $\begin{array}{c}5.50 \\
(2.14)\end{array}$ \\
\hline
\end{tabular}

\section{Conclusions}

In this experiment we studied learners who could be characterized by low prior knowledge and with high learning 
goals set by the expectation of a comprehension test. Concerning our first research question, we found that the reading order is a strong mediating factor that directly affects cognitive load and comprehension. Participants following a high text coherence reading order suffered less cognitive load and also achieved a better learning at situational level than those following a low text coherent reading order. Second, the effects of the number of links were not significant. In contrast to the predictions of DeStefano and LeFevre [8], our results did not show any evidence of an increase on cognitive load when more links were presented, nor an effect on learning. Thirdly, we included link suggestions -based on semantic similarity- and examined the influence on cognitive load and comprehension. The results of analysis with regards to suggestions were only partly analysed (and partly reported here), because of low number of subjects. However, participants in the support condition followed as predicted a high coherent reading order and achieved better comprehension outcomes. In other words, when low prior knowledge learners receive navigation support based on methods that calculate the semantic similarity between connected pages in the hypertext, they are able to develop a more coherent reading order and construct a better situation model of the learned material. We also did not found a reduction on cognitive load when link support was offered (see [18]).

\section{EXPERIMENT 2: MONITORING SKILL AND LINK SELECTION}

In a second experiment we tried to find an explanation for the differences in selected reading order that participants in Experiment 1 showed. In the previous experiment all participants were mostly LPK readers and were stimulated by the instruction to set a relatively high learning goal. However, not all participants selected a coherent reading order. The reason for this difference can be due to differences in the comprehension monitoring skills that readers have. Maybe only readers with high monitoring skills are able to select and follow a semantic high coherent reading order strategy.

The concept of monitoring skills for comprehension plays a central role in the model of self-regulated learning (SRL) proposed by Winne [13, 14]. The model considers readers as active participants in the learning process, as reflected by the different cognitive and metacognitive activities in which readers engage in a cyclic and recursive manner. Based both on task conditions (e.g., an assigned goal) and on cognitive conditions (e.g., how much prior knowledge of the topic readers possess), readers set a goal or standard to guide learning. Once readers have set a goal, they apply study tactics to attain their goal. During the execution of these processes, readers monitor their comprehension and the effectiveness of the strategies used. A perceived mismatch between the goal and current comprehension could lead to adjustments to the learning goal or to the study strategies used. An accurate monitoring of comprehension is necessary to detect a mismatch between the comprehension induced by the strategies applied and reader's learning goal. Although Winne's SRL model was first developed for traditional linear text, it has been successfully applied to the study of reading in hypertext (e.g. [19]). From this perspective, hypertext readers with higher monitoring skills check regularly the effects on comprehension derived from the use of particular link selection strategies, i.e. a higher comprehension related to the coherence strategy. Thus, provided that a high learning goal has been set, readers with higher monitoring skills will carry out optimal strategies for link selection. For instance, the strategy that tries to select links that provide the highest text coherence. Readers with low monitoring skills, by contrast, might not detect the comprehension problems associated to non-optimal link selection strategies. In other words, an inability to accurately monitor comprehension might prevent readers from using a more appropriate strategy. The fourth research question of this paper is whether there is a direct relation between monitoring and link selection strategies, two factors which have a robust impact on comprehension.

\section{Method}

\section{Participants}

Thirty-nine students from the University of Colorado from an introductory psychology class participated in the experiment. As in experiment 1, we were interested in testing our hypotheses on low prior knowledge readers. For this reason, we selected participants from a larger sample, based on their scores in a Prior Knowledge questionnaire. Only those with a score below percentile 25 (3 or less, out of 10 questions) were considered for these analyses (for more details see [20]).

\section{Design and Measures}

Coherence of the Selected Reading Order. We followed the same procedure as in experiment 1 to group participants according to the degree of coherence of the reading order of their navigational path (see below for more details).

Monitoring skill test. A “judgment of learning task" was used to assess the participants' monitoring skill. For each page read of the hypertext, participants judged their perceived comprehension by answering the question "How likely is it that you will be able to correctly answer a test question about the section you just read in about 25 minutes?' 0 (definitely won't be able to), 10 (10\% sure I will be able to), 20..., 30.., 100 (definitely will be able to)". For each participant, a gamma correlation between judgments and test performance across pages was computed (for details on the comprehension test used see below). The Gamma correlation represents the degree to which a person's judgments correlate with his or her own test performance across text sections. The correlation ranges from -1 to +1 , with correlations near or below 0 indicating poor accuracy. This is exemplified in two possible cases: either a reader thought she will be able to correctly answer most of the questions and then failed most of them, or she thought she will not correctly answer most of the test and then succeed on it. A correlation of 1 indicates a perfect match between participants' judged comprehension during reading and their actual comprehension as measured by post-test questionnaires. Again, this corresponds to two possible scenarios: either a reader thought she will be able to answer most of the questions and then succeed on most of them, or she thought she will not correctly answer most of the test and then failed on it. 
Comprehension Outcomes. To assess comprehension at a situation model level, we constructed 10 inference questions with three response options. This type of questions required participants pull together information contained in at least two different pages. In addition, we used 22 multiple-choice text-based questions that targeted information that occurred in a single page, covering most of the hypertext information.

\section{Materials}

We adapted an expository text on atmospheric pollution for use in a hypertext format. The text was 4,033 words long (including section titles) and was divided in 27 hypertext pages. One hypertext page was presented on the screen, and after finishing reading it, participants could choose between only two possible links. These two links corresponded to the section with the highest coherence and to the section with the lowest coherence with the previously read text. Coherence between texts was computed using LSA as in experiment 1. Links were presented one below the other. The position of the high and low coherence links was randomized across selections. Participants were not aware of the distinction between links. Each text section was presented only once and it was not possible to backtrack and reread it.

\section{Procedure}

Participants started the experiment filling in the prior knowledge questionnaire. They were then instructed on how to use the hypertext system and were asked to read the text without time restrictions. The instructions stressed that they would have to read all sections of the text but that they could choose the order in which to read them. As in experiment 1 , participants were instructed to set a high learning goal for the reading session. Specifically, participants were told to "read the sections and select the order carefully because you will have to answer some questions about the content after reading the entire text." The reading procedure was as follows: first, participants were presented with an introductory hypertext page with an overall description of the text. After reading that section, participants had to press a button announcing that they had finished reading it. They then performed a judgment of learning for that section. Next, a new screen appeared presenting two links pointing to unread pages. Here, participants had to click on a link to read one of the sections. The selected text was presented on the screen with a button announcing the completion of the reading. Once this button was pressed a new page with 2 links to select appeared. When all text sections were read, participants went to the comprehension-testing phase.

\section{Results}

\section{Monitoring Skill and Coherence of the Selected Reading Order}

Mean gamma correlations for our sample was 0.28, $S D=0.3$, which indicates that participants had just a moderately accurate monitoring skill. This result is in line with previous research using analogous methodologies for the assessment of monitoring skill [21]. In order to test our fourth research hypothesis concerning a possible causal relationship between monitoring skill and the coherence of the reading order, we performed a linear regression analysis with monitoring skill as the only predictor and LSA cosines as dependent variable. The predictor accounted for a modest part of the variance in LSA cosines $\left(R^{2}=.14\right)$, which was significant, $F(1,36)=5.56, p<.05$. High monitoring skills predicted the selection of a high coherent reading order.

\section{Monitoring Skill, Coherence of the Selected Reading Order, and Comprehension}

We also analyzed the effect of monitoring skill and coherence of the reading order on comprehension. These two predictor variables were entered into a multiple regression analysis, including scores on the inference questions as dependent variable. Monitoring skill did not predict inference scores, $F<1$, but LSA cosines $\mathrm{did}, R^{2}=.13$, $F(1,35)=2.03, p<.05$. High LSA cosines -indicating a selection of a coherent reading order- positively predicted scores on inference questions.

\section{Conclusions}

As in Experiment 1, this second study focused on readers with low prior knowledge instructed to attain a high learning goal. Concerning the fourth research question, we found that high monitoring skills predicted the selection of a more coherent reading order in hypertext. After setting a high study goal (as requested by the instructions), participants monitor their comprehension against their goal while reading, and regulate their reading behaviour to adjust actual comprehension to their learning goal [14]. Apparently readers who are able to detect on time comprehension difficulties impeding them to attain their learning goal perform more successful link selections leading to high text coherence.

With regard to the second aim of this study, results revealed that only the coherence of the reading order followed by participants (measured by LSA cosines) and not monitoring skill per se had a positive direct impact on comprehension at the situation model level (as measured by inference questions).

\section{GENERAL DISCUSSION AND CONCLUSION}

Although learning with hypertext is nowadays extremely popular, is still not clear whether comprehension and learning is better in comparison to traditional learning methods. One problem related with hypertext is the increase on cognitive load that learners suffer while learning with hypertext. The main reason for the increase on cognitive load is the fact that learners have to decide by themselves what is the best link path to follow [8]. On the other hand, giving navigation support in the form of link suggestions based on semantic similarity helps users in navigation and learning (e.g. $[10,22]$.

In the first experiment we reported the effects of selected reading order and the number of links on cognitive load and comprehension. Regarding our first research question, we found that the reading order is a strong mediating factor that directly affects cognitive load and comprehension. Participants following a high text coherence reading order suffered less cognitive load and achieved a better learning at situational level than those following a low text coherent reading order. Regarding our second research question and in contrast to the predictions of [8], our results did not show any evidence of an increase on cognitive load when more links were presented. On the other hand, our third hypothesis 
was confirmed. As reported in detail in [18], participants that received link suggestions followed a high coherent reading order and achieved better comprehension outcomes at situation model level. In other words, when low prior knowledge learners receive navigation support based on methods that calculate the semantic similarity between connected pages in the hypertext, they are able to develop a more coherent reading order and construct a better situation model of the learning material.

In the second experiment we explored our fourth research question: the role of monitoring skills in the selected reading order and comprehension. We found that high monitoring skills predicted the selection of a more coherent reading order in hypertext. In relation with comprehension, we found that the coherence of the reading order followed by participants and not monitoring skill per se had a positive direct impact on comprehension at the situation model level. This data seem to reflect a clear mediation model: monitoring skills impact comprehension during reading through diverse adjustments of the reading behaviour due to the use of tactics and strategies. A particular kind of adjustment that takes place after setting a high learning goal is selecting hyperlinks based on semantic similarity between the text just read and the links to select. This adjustment affects comprehension because it leads to changes in the coherence of the hypertext [7,23].

The two experiments reported here stress the importance of selecting a coherent reading order in hypertext in order to improve reader's comprehension. Both experiments also suggest two ways to help readers select hyperlinks based on the semantic coherence between sections. Experiment 1 corroborates the usefulness of link suggestions (see [18]) whereas Experiment 2 suggests that improving reader's monitoring skills might induce them to follow a more coherent reading order. Future research might explore techniques to improve readers' monitoring skills with the aim of facilitating students' selection of an optimal reading order. In this sense [24], describe a hypertext system that analyses in real time the reading sequence of students, and prompts them to assess their comprehension whenever transitions between low semantically related sections are made. Thus, this system aims to stimulate monitoring of comprehension during reading, under the assumption corroborated in our Experiment 2 - that better monitoring is related to more optimal link selection strategies. Such a system may benefit by incorporating a recent technique to improve monitoring skill, which consists on instructing students to judge their understanding of specific ideas (e.g. regarding a text of elementary statistics: 'How well will you be able to recall the definition of ordinal measurement on an upcoming test?') and not of an entire section of the text [21]. Readers are required to attempt to recall the definition before the actual judgment of comprehension, and are subsequently provided with feedback on their response. Thus, readers know immediately if they really understood the text at the level they thought. We might expect that an advancement on readers' calibration accuracy would reflect on an improvement on their reading decisions in hypertexts (e.g. selection of a coherent reading order, allocation of study time...). Further research will be required to evaluate this issue.
In addition, the results from the reported experiments suggest several challenges for researchers who are currently examining the role of strategic behaviour in hypertexts. Future research might address how link suggestions and monitoring prompts impacts readers' use of other key hypertext strategies besides the selection of reading order, such as the selection of sections and the allocation of study time. Shapiro and Niederhauser [25] have suggested that self-regulation may well be related to the process of deciding which sections to read (and which to ignore), an important task that can also affect comprehension [26]. This is exactly the reason why we devised link suggestions based on semantic criteria, so readers can easily avoid hypertext sections not relevant for their learning task. And this suggestion did lead us to hypothesize and find an improvement in navigation and learning performance for LPK when link suggestions based on semantic criteria are offered elsewhere [22].

\section{REFERENCES}

[1] Chen SY, Fan J, Macredie RD. Navigation in hypermedia learning systems: Experts vs novices. Comput Hum Behav 2004; 22: 25166.

[2] Kintsch W. The role of knowledge in discourse comprehension: A construction integration model. Psychol Rev 1988; 95: 163-82.

[3] Kintsch W. Comprehension: A Paradigm for Cognition. New York: Cambridge University Press 1998.

[4] Britton B, Gülgöz S. Using Kintsch's computational model to improve instructional text: Effects of inference calls on recall and cognitive structures. J Educ Psychol 1991; 83: 329-45.

[5] McNamara DS, Kintsch E, Songer N, Kintsch W. Are good texts always better? Interactions of text coherence, background knowledge, and levels of understanding in learning from text. Cognit Instruct 1996; 14: 1-43.

[6] Salmerón L, Cañas JJ, Kintsch W, Fajardo I. Reading strategies and hypertext comprehension. Discourse Process 2005; 40: 171-91.

[7] Salmerón L, Kintsch W, Cañas JJ. Reading strategies and prior knowledge in learning with hypertext. Mem Cognit 2006a; 34: 1157-71.

[8] DeStefano D, LeFevre JA. Cognitive load in hypertext reading: A review. Comput Hum Behav 2007; 23: 1616-41.

[9] McNamara DS, Shapiro AM. Multimedia and hypermedia solutions for promoting metacognitive engagement, coherence and learning. J Educ Comput Res 2005; 33: 1-29.

[10] Salmerón L, Kintsch W, Cañas JJ. Coherence or interest as a basis for improving hypertext comprehension. Inf Design J 2006b; 14: 45-55.

[11] Puerta Melguizo MC, Van Oostendorp H, Juvina I. Predicting and solving Web navigation problems: Proceedings of the Hypertext 2007: Eighteenth ACM Conference on hypertext and hypermedia; September 9-12, 2007. Manchester, United Kingdom; pp. 47-48.

[12] Foltz PW, Kintsch W, Landauer TK. The measurement of textual coherence with Latent Semantic Analysis. Discourse Process 1998; 25: 285-307.

[13] Winne PH. Inherent details in self-regulated learning. Educ Psychol 1995; 30: 173-87.

[14] Winne PH. Self-regulated learning viewed from models of information processing. In: Zimmerman B, Schunk D, Eds. Selfregulated learning and academic achievement: Theoretical perspectives. Mahwah, NJ: Lawrence Erlbaum Associates 2001; pp. 153-89.

[15] Anderson JR. Cognitive Psychology and its implications. $6^{\text {th }}$ ed. New York: Worth Publishers 2005.

[16] Brünken R, Plass JL, Leutner D. Direct measurement of cognitive load in multimedia learning. Educ Psychol 2003; 38: 53-61.

[17] Boeree G. General Psychology. Shippensburg University; 2003 available from: http://webspace.ship.edu/cgboer/genpsy.html

[18] Madrid RI, Van Oostendorp H, Puerta Melguizo MC. The effects of the number of links and navigation support on cognitive load and learning with hypertext: The mediating role of reading order. Comput Hum Behav 2009; 25: 66-75. 
[19] Azevedo R, Cromley JG. Does training on self-regulated learning facilitate students' learning with hypermedia? J Educ Psychol 2004; 96: 523-35.

[20] Salmerón L, Kintsch W, Kintsch E. Self-regulation and link selection strategies in hypertext. Discourse Process 2010; in press.

[21] Dunlosky J, Lipko AR. Metacomprehension: A brief history and how to improve its accuracy. Curr Direct Psychol Sci 2007; 16: 228-32.

[22] Van Oostendorp H, Juvina I. Using a cognitive model to generate web navigation support. Int J Hum Comput Stud 2007; 65: 887-97.
Budd D, Whitney P, Turley KJ. Individual differences in working memory strategies for reading expository text. Mem Cognit 1995; 23: 735-48.

[24] Puntambekar S, Stylianou A. Designing navigation support in hypertext systems based on navigation patterns. Instruct Sci 2005 33: 451-81.

[25] Shapiro AM, Niederhauser D. Learning from hypertext: research issues and findings. In: Jonassen DH, Ed. Handbook of Research on Educational Communications and Technology, $2^{\text {nd }}$ ed. Mahwah, NJ: Lawrence Erlbaum Associates 2004; pp. 605-20.

[26] Lawless KA, Kulikowich JM. Understanding hypertext navigation through cluster analysis. J Educ Comput Res 1996; 14: 385-99.

(C) Melguizo er al.; Licensee Bentham Open.

This is an open access article licensed under the terms of the Creative Commons Attribution Non-Commercial License (http: //creativecommons.org/licenses/by$\mathrm{nc} / 3.0 /$ ) which permits unrestricted, non-commercial use, distribution and reproduction in any medium, provided the work is properly cited. 